\title{
Diagnóstico de la compresión lectora en los bachilleres de la unidad educativa Jhon F. Kennedy en la provincia de Santa Elena
}

\author{
Katherine Julissa González Figueroa \\ katherine.gonzalezfigueroa@upse.edu.ec
}

Damnesa Selena Muñoz Mejillones damnesa.munozmejillones@upse.edu.ec

\author{
Mercedes Magdalena Reyes Baque \\ mercedes.reyesbaque@upse.edu.ec \\ Denisse Estefania Tomalá Guzmán \\ denisse.tomalaguzman@upse.edu.ec
}

Eliza Daly Tigrero González eliza.tigrerogonzalez@upse.edu.ec Universidad Estatal Península de Santa Elena La Libertad- Ecuador

\section{RESUMEN}

La presente investigación se planteó como objetivo principal: Diagnosticar la comprensión lectora en los bachilleres de la Unidad Educativa Jhon F. Kennedy del cantón Salinas, provincia de Santa Elena. El estudio se enmarcó en un diseño transversal con enfoque cuantitativo y metodología descriptiva. La muestra estuvo conformada por 43 estudiantes cuyas edades oscilan entre los 15 a 16 años. Para la recolección de datos se diseñó una encuesta con preguntas semiestructuradas asociadas a la comprensión lectora, de igual manera, fueron dirigidas a las técnicas de lectura y al tiempo de ocio orientado a la lectura. Para garantizar la confiabilidad del instrumento se aplicó el Coeficiente de Cronbach determinando un valor de 0,886. Los principales resultados demuestran que, el $21 \%$ de los estudiantes presentan un nivel alto de comprensión lectora, el $51 \%$ un nivel intermedio y, el $28 \%$ un nivel bajo. Se concluye que, el tiempo de lectura y la comprensión de los textos varían, según los intereses, las capacidades y habilidades cognoscitivas de los estudiantes.

Palabras clave: hábitos; técnicas; comprensión lectora; estudiantes 


\title{
Diagnosis of reading comprehension in high school graduates of the Jhon F. Kennedy educational unit in the province of Santa Elena
}

\begin{abstract}
The main objective of this research was to diagnose reading comprehension in high school students of the Jhon F. Kennedy Educational Unit in Salinas, Santa Elena province. The study was framed in a transversal design with a quantitative approach and descriptive methodology. The sample consisted of 43 students between 15 and 16 years of age. For data collection, a survey was designed with semi-structured questions associated with reading comprehension, as well as reading techniques and leisure time oriented to reading. To guarantee the reliability of the instrument, Cronbach's Coefficient was applied, determining a value of 0.886 . The main results show that $21 \%$ of the students present a high level of reading comprehension, $51 \%$ an intermediate level and $28 \%$ a low level. It is concluded that reading time and text comprehension vary according to the students' interests, abilities and cognitive skills.
\end{abstract}

Keywords: habits; techniques; reading comprehension; students

Artículo recibido: 15 noviembre. 2021 Aceptado para publicación: 10 diciembre 2021 Correspondencia: katherine.gonzalezfigueroa@upse.edu.ec

Conflictos de Interés: Ninguna que declarar 


\section{INTRODUCCIÓN}

El presente Artículo es el resultado de la investigación que se desarrolló en la asignatura de Diseño de Proyectos Educativos que se imparte en el sexto semestre de la Carrera de Educación Inicial de la Universidad Estatal Península de Santa Elena, donde surge la necesidad de Diagnosticar la comprensión lectora en los bachilleres de la Unidad Educativa Jhon F. Kennedy del cantón Salinas, provincia de Santa Elena-Ecuador.

Las motivaciones que dan origen a esta investigación surgen de la importancia que tiene el ejercicio de la lectura en los estudiantes, puesto que, como actividad cognoscitiva estimula el desarrollo de la percepción, la concentración, la imaginación, la creatividad; que, como habilidades del pensamiento son importantes en el contexto académico en el que se encuentran inmersos los discentes. Con este resultado, se planifican acciones que fortalecen los hábitos de la lectura y beneficia el aprendizaje de los estudiantes.

El propósito de la lectura es entender y darle sentido al texto, es decir, lograr que el discente adapte lo leído a los saberes que ya tiene, de aquí que, la comprensión lectora se vincule con la visión que cada uno tiene sobre el mundo y de sí mismo, puesto que, ante un determinado contenido, los educandos no llegan a una sola interpretación del escrito. A su vez, la lectura posibilita que los estudiantes desarrollen aptitudes cognoscitivas que les permitirán construir nuevas estrategias o habilidades como: evaluar, seleccionar y descartar ideas en función de lo analizado.

Según, Solé (1997) "un lector activo procesa información en diferentes sentidos aportando desde su conocimiento y en conjunto con su capacidad de crear inferencias al entendimiento del texto"; además, se debe mantener alerta a lo largo del proceso aportando con una buena interpretación y, si es objetivo, está capacitado para construir competencias de aprendizaje; es decir, debe ser capaz de seleccionar, procesar y recordar contenidos de modo comprensible, de tal manera que, pueda aplicarlos en contextos demostrativos como: educativos y sociales. Por otro lado, cada lector otorga un significado a la lectura, coordinando información que obtiene de distintas fuentes y doctrinas que posee, utilizando como base las habilidades y estrategias que le ayuden a construir sus capacidades cognitivas.

Una aproximación al concepto de la comprensión lectora lo aporta Luque (2005), que señala, "es importante conocer cuáles son los componentes necesarios y los pasos a seguir para llegar a ésta"; por tanto, es sustancial definir qué es "leer". Para Cruz (2004) "Leer 
es un proceso de interacción entre lector y el texto, proceso, mediante, el cual intenta satisfacer los objetivos que guían su lectura".

Para Snow (2001), "la comprensión lectora es el proceso simultáneo de extracción y construcción del significado a través de la interacción e implicación con el lenguaje escrito", además, busca que el lector identifique ciertos elementos de un texto definido donde éste sea capaz de analizar las situaciones que se presentan, decodificando, traduciendo e interpretando, mediante, el lenguaje oral, el cual, permite al lector localizar palabras, fácilmente. Al estudiante cuando se relaciona con el texto le permite tener la oportunidad de aprender y contrastar con lo que ya sabe, esto debe ir unido a la interpretación, es decir, que el lector tenga la capacidad de predecir el texto.

Según Jiménez Pérez (2014), "la competencia lectora es la capacidad de comprender, usando aquellas formas del lenguaje escrito requeridas por la sociedad o valoradas por la persona". Es una habilidad, la cual consiste en manejar saberes técnicos, donde el lector se basa en lo que quiere decir como reflexión final, esto es un poco complejo y concreto, en consecuencia, pasa de implementar las habilidades a utilizar sus capacidades mentales para la interpretación de los textos y su opinión del contenido antes leído como reconstruir las múltiples ideas del texto, es así que, se logra verificar si el lector cuenta con las destrezas necesarias para establecer sus etapas de competencia a nivel educativo, considerando la amplitud de argumentos que puede procesar, la fluidez de lectura y la interpretación variada de textos.

\section{Ejes de la competencia lectora}

Aprender a leer

Es el desarrollo de la capacidad y destreza específica para que los estudiantes lleguen a la adquisición de las habilidades fundamentales en la cultura letrada actual y en el ambiente educativo.

Es un recurso fundamental para el aprendizaje de los

Leer para aprender estudiantes y la ampliación del conocimiento en el aula y fuera de ella, además, de captar la información que le permitirá realizar inferencias sobre el texto y analizarlo de manera crítica.

Aprender a disfrutar Es el logro e interpretación de la lectura que poseen los de la lectura estudiantes, independientemente de la intención o características del texto leído

Nota 1: Solé, Estrategias de Lectura, (1998). 
La tabla muestra que los ejes de la competencia lectora permiten a los estudiantes entender, comprender y reflexionar sobre textos escritos para alcanzar sus propias metas académicas, desarrollando el conocimiento, las habilidades y destrezas que los educandos adquieren en su desempeño educativo. Cabe recalcar, que las mismas son esenciales en el proceso de enseñanza-aprendizaje influyendo y fomentando el gusto por la lectura, posteriormente, alcanzarán a inferir y decodificar, de manera independiente, adquiriendo velocidad y fluidez lectora para el análisis de contenidos a manera crítica y coherente.

La comprensión lectora es una de las habilidades principales para que los estudiantes desarrollen las actividades de aprendizaje, por medio de éstas, se consiguen realizar los análisis, identificando las ideas primarias y secundarias de un texto, esto permite, generar conclusiones sobre lo leído. Por tanto, la comprensión lectora proporciona al lector la habilidad para construir conceptos y la capacidad de entenderlos y aplicarlos a la resolución de problemas académicos.

Desarrollar la comprensión lectora es una tarea difícil para los estudiantes, porque, requiere de habilidades cognitivas superiores que los educandos no han fortalecido todavía; sin embargo, es necesario ya que posibilita la capacidad de pensar, despertar aficiones e intereses, imaginar lo que se está leyendo; potencia la gran formación estética. Comprender textos es una habilidad fundamental para el crecimiento personal de los dicentes, contribuye a aumentar el vocabulario fortaleciendo el pensamiento creativo y el razonamiento abstracto.

El hábito de la lectura es la capacidad que cada estudiante puede desarrollar, mediante, ejercicios académicos, así mismo, vinculando la decisión de aprendizaje autónomo en la práctica de estrategias que ejecuta de modo consciente. La lectura conlleva a un comportamiento estructurado, donde los educandos adquieren la competencia lectora, además, comprende la repetición frecuente del acto de leer, el conjunto de destrezas y habilidades implicadas en esta actividad, gracias a su interacción y al progresivo dominio de sus mecanismos. (Armella \& López De Llergo, 2004)

Cuando se fomenta el hábito de la lectura, éste contribuye a que los estudiantes refuercen sus experiencias de aprendizaje para educarse durante toda la vida, también, se fortalece el enriquecimiento de su intelecto, la ampliación del vocabulario y la posibilidad de mejorar en el ámbito educativo. Por ende, los padres y maestros influyen en la formación del hábito lector, inculcando a los educandos los beneficios de leer; un leyente no es aquel 
que analiza cantidades de libros o dedica tiempo a dicha práctica, sino, el que llega a buenos resultados de comprensión e inquisición, es decir, que deja enseñanzas duraderas y explícitas en la vida del estudiante.

Por otra parte, las técnicas de lectura son procesos que estimulan la reflexión para una mejor interpretación y generalización, se caracterizan por adaptar la manera de leer al propósito del lector, adquiriendo conocimientos para el aprendizaje, pues, existen diversos formatos dotados por símbolos o letras que hacen posible la comprensión de los materiales escritos utilizados en actividades académicas (Hoyos Flóres \& Gallegos, 2017, págs. 23-27). Al profundizar una lectura se utilizan técnicas como la convencional que está enfocada en la velocidad lectora, la misma que facilita el análisis de manera “explícita” al lector y, proporciona pautas para el desarrollo de habilidades, considerando así, la lectura como un conjunto de actividades críticas, donde se evalúa la interacción existente entre el pensamiento, el lenguaje, la transición y el texto. (Alvarez Alberdi, 1993, págs. 83-91)

Es importante señalar, que las técnicas de lectura proporcionan un proceso semántico para comprender lo leído. El estudiante lee, mediante, el proceso léxico y sintáctico resaltando lo más significativo de un texto, evaluando, de manera crítica, los puntos de vista de forma imparcial, elaborando opiniones sobre los hechos con validez y fundamentos con la finalidad de adquirir nuevos conocimientos. (Solé, 2012, pág. 43) Las técnicas de lectura buscan guiar a los lectores, evitando leer contenido innecesario con el propósito de que el estudiante no lo emplee posteriormente, realizando una revisión detallada del contenido para identificar los objetivos y el propósito del mismo. (Tovar Cabañas, 2009, págs. 3878)

Las técnicas de lectura requieren una variedad de destrezas específicas que ayudan a comprender lo que se lee, así mismo, estimulan y aumentan la capacidad intelectual, también, ayudan a reflexionar y comunicarse mejor, pues, se adquiere una nueva perspectiva de la realidad. 


\section{Técnicas de lectura}

\begin{tabular}{ll}
\hline \multirow{2}{*}{$\begin{array}{l}\text { Lectura } \\
\text { secuencial }\end{array}$} & $\begin{array}{l}\text { Permite a los estudiantes comprender de qué se está hablando en } \\
\text { la lectura, la idea principal del texto y cómo surgen los } \\
\text { acontecimientos. Es decir, que leen en su tiempo individual desde } \\
\text { principio a fin, sin repeticiones u omisiones de la lectura. }\end{array}$ \\
\hline $\begin{array}{l}\text { Lectura } \\
\text { intensiva }\end{array}$ & $\begin{array}{l}\text { Implica que cada estudiante interprete las intenciones del autor } \\
\text { centrándose en los detalles con la finalidad de obtener } \\
\text { información específica. }\end{array}$ \\
\hline $\begin{array}{l}\text { Lectura } \\
\text { puntual }\end{array}$ & $\begin{array}{l}\text { Los estudiantes leen las partes importantes, es decir, se enfocan } \\
\text { en aquellos contenidos del texto que les resultan interesantes. }\end{array}$ \\
& $\begin{array}{l}\text { Esto, les permite ahorrar tiempo, de este modo, se puede acceder } \\
\text { a mucha información en poco tiempo. }\end{array}$ \\
\hline \multirow{2}{*}{$\begin{array}{l}\text { La pectura } \\
\text { inferencial }\end{array}$} & $\begin{array}{l}\text { atención en los aspectos más relevantes, esta técnica de lectura es } \\
\text { importante para evaluar la comprensión de contenidos, implica } \\
\text { leer una parte y se infiere el resto. }\end{array}$ \\
\hline
\end{tabular}

Nota 2: Madero Suárez \& Gómez López, (2013).

Según, lo mencionado, la comprensión lectora se enfoca en estrategias que son importantes para el proceso de la lectura como se explica a continuación:

Las estrategias de aprendizaje se muestran como una toma de decisiones sobre la selección y uso de procedimientos que facilitan una lectura activa, intencional, autorregulada, competente; las destrezas metacognitivas previas al repaso se priorizan, mediante, acciones importantes: identificar y determinar el género discursivo con la finalidad de activar conocimientos previos, generar preguntas que son contestadas con la interpretación del texto. Los tipos de técnicas de corrección son: releer, parafrasear y resumir entidades textuales adecuadas, cuando el estudiante es consciente de la falta de comprensión. (Gutierrez Braojos \& Salmerón Pérez, 2012, págs. 183-202)

Es necesario que, el estudiante sepa qué debe hacer, esta condición remite a los objetivos que presiden la lectura; siendo un factor que contribuye a encontrar un contenido interesante de un determinado material textual, por ende, consiste en retos utilizar textos no conocidos, teniendo en cuenta las posturas lectoras. Una mala comprensión de significados afecta, directamente, al nivel de estudios, siendo necesario un mayor esfuerzo e inversión de tiempo para obtener resultados excelentes, por ello, las técnicas 
de comprensión lectora inician desde los procesos de adiestramiento de mejoras del entendimiento de los conceptos descritos en un argumento, la combinación de las diferentes técnicas y estrategias son sumadas a la capacidad individual para la asimilación de escritos. (Rivas Cedeño, 2015, págs. 47-61)

Conseguir vivencias óptimas en conformidad a la lectura desarrollan habilidades, capacidades, competencias; es aquí, donde la enseñanza del período libre y la comprensión lectora juegan un papel importante, puesto que, los estudiantes prefieren realizar otras actividades como: ver la televisión, entrenar deportes, utilizar el ordenador, el celular, oír canciones, leer, jugar o pasar muchas horas en cosas innecesarias. Una forma del tiempo de ocio posibilita conformar, modificar y motivar a los educandos, involucrando el momento necesario en guiarlos y comprometerlos a que destinen un espacio diario para leer, además, reduce el estrés, de forma que, aumenta la función cerebral, formándolos empáticos, imaginativos, creativos; mejorando su vocabulario, la comprensión lectora, tema de este estudio.

Además, la lectura que se realiza, libremente, durante el tiempo de ocio, potencia las capacidades cognitivas precisas para los estudiantes, tanto durante su proceso de formación como en su desarrollo profesional. Por otra parte, la identificación de las buenas prácticas de pasatiempos se hace cada vez más relevante para la intervención en la mejora de la calidad educativa y el perfeccionamiento integral de los discentes, es preciso conocer qué implicación tiene el empleo del descanso sobre la vida académica específicamente, analizar el papel que ocupa la lectura en el éxito académico de los educandos para conseguir una buena comprensión y competencia lectora siendo uno de los principales objetivos educativos. (García, 2013, pág. 133)

La comprensión lectora es importante en las actividades formales e informales de los estudiantes, en este caso, debido al desinterés de aprender o conocer palabras técnicas y su significado conceptual, el mismo que, puede ser difícil comprender, especialmente, cuando se genera una explicación sobre un tema determinado y en definir un nuevo vocabulario. De allí que, la lectura comprensiva se realiza con el fin de poder implementar mejoras en este proceso y, a su vez, los factores que influyen en la comprensión de la misma como el lector, el texto, los conocimientos previos que poseen y las formas que utilizan para realizar dicha gestión. 
Según Díaz \& Hernández (2010), "las estrategias de aprestamiento son aquellas que permiten realizar una preparación previa para la lectura de un texto, están relacionadas con el propósito de leer y con las actividades de planificación para afrontar el proceso de comprensión”. De manera que, leer en sí, es una destreza de aprendizaje y en la misma se involucran los hábitos que se encuentran ligados a las actitudes adaptativas de cada estudiante en su rutina de autoeducarse; es aquí, donde el hábito de los educandos es leer textos académicos típicos en correspondencia a varias asignaturas; es gradual y guiado, especialmente, si se consideran las dificultades existentes.

Dado que la educación secundaria debe asegurar que los estudiantes comprendan la importancia de adoptar una actitud más positiva y llevar a cabo el proceso de lectura con un mayor nivel de conciencia para convertirse en lectores capaces de desarrollar una inferencia coherente y, a su vez, el entendimiento por interés propio, los ayudará a ser participativos ante los demás; generalizando e integrando un conjunto de proposiciones que comprenden una información explícita. Entonces, comprender se precisa como la capacidad de realizar un aprendizaje variado de conocimientos que estimulen el pensamiento, como son explicar, demostrar, ejemplificar, generalizar, volver a presentar el tema de otra manera, entre otras y para conseguirlo se debe leer con claridad respetando todas las reglas.

Según Mantilla (2008), “el primer paso para lograr el deleite hacia la lectura es apreciar el libro como objeto, existe una relación entre la apariencia de un libro y la atracción que influye sobre sus posibles lectores". Con ello, se logrará estimular el uso de herramientas, tales como libros y diccionarios en los que se realizan listas de palabras complejas o combinaciones, entre ellas para conectar conjeturas y consigan ser comprendidas, por último, realizar un resumen con las ideas principales del mismo. Siendo así, una mejora de las condiciones sociales y humanas de cada estudiante, puesto que, leer permite el desarrollo del pensamiento reflexivo y progresivo de los aspectos cognitivos del cerebro. Ante lo expuesto, la presente investigación se plantea como objetivo principal: Diagnosticar la comprensión lectora en los bachilleres de la Unidad Educativa Jhon F. Kennedy del cantón Salinas, provincia de Santa Elena-Ecuador.

\section{ESTRATEGIAS METODOLÓGICAS O MATERIALES Y MÉTODOS}

El presente Artículo se enmarca en un diseño instrumental con enfoque metodológico cuantitativo, porque, de acuerdo con León \& Montero (2003), pertenecen a esta categoría 
"todos los estudios encaminados al desarrollo de pruebas, incluyendo el diseño como el estudio de las propiedades psicométricas de los mismos" (pp.799), mediante, el análisis cuantitativo de los datos obtenidos por la triangulación de tres fuentes de información: estudiantes, docentes y expertos.

Es una investigación de campo porque orienta la práctica hacia un cambio, identificando procesos para conocer y actuar ante la situación, es decir, que va de lo general a lo específico, a su vez, pretende dar respuesta a la problemática, persiguiendo el desarrollo y contrastación de diferentes explicaciones en un marco específico definido, comprendiendo e involucrando a los estudiantes del 2do Bachillerato General Unificado (BGU).

El estudio es de alcance descriptivo porque se fundamenta sobre realidades de hechos, y su característica esencial es la de presentar una interpretación correcta; para que la investigación sea detallada, su preocupación primordial radica en descubrir algunas características fundamentales de conjuntos homogéneos de fenómenos, utilizando criterios sistemáticos que permitan orientar de manifiesto su estructura o comportamiento, de esta forma, se consiguen obtener los análisis que determinan a la realidad estudiada. (Sabino, 1992, pág. 216)

Para Hernández Sampieri (2003), "la investigación no experimental se divide tomando en cuenta el tiempo durante la recolección de los datos", por este medio, se define el diseño transversal; el propósito es describir variables. Se fundamenta en planteamientos e hipótesis correlacionales, del mismo modo, que busca evaluar vinculaciones causales. Por ello, es una investigación observacional que permite darle sentido al Artículo desde que se muestra el problema y se busca la solución, de manera que, se solventen las complicaciones del mismo para esclarecer las ideas durante el proceso investigativo.

La muestra estuvo conformada por 43 estudiantes con edades comprendidas entre 15 a 16 años, de la Unidad Educativa Jhon F. Kennedy del cantón Salinas. En lo que respecta al género se cuenta con 21 hombres y 22 mujeres del Segundo año del Bachillerato General Unificado.

Para la recolección de datos se elaboró una encuesta con 24 preguntas con su respectiva escala de Likert la misma que dependía de los siguientes valores: nunca (1), raramente (2), ocasionalmente (3), frecuentemente (4), muy frecuente (5). 
El instrumento se diseñó con los siguientes ejes temáticos: el primero, Comprensión lectora, con 10 preguntas relacionadas a la interpretación, decodificación y síntesis para adecuar la comprensión de los contenidos leídos; la segunda, Técnicas de lectura; presentando 8 propuestas de incógnitas en constancia de diferentes formas para seleccionar, de manera correcta, los argumentos de un texto determinado y, la tercera: Tiempo de ocio; con 4 consultas acerca del momento apropiado para dedicarlo a algún libro o reforzar el repaso del mismo. Todos estos indicadores, dirigidos a los estudiantes del 2do "BGU" de la Unidad Educativa Jhon F. Kennedy.

La validez del instrumento se basó en el juicio del experto, éste se define según, Kane (2006), como "una aprobación basada en argumentos que implica dos pasos: la especificación de las interpretaciones propuestas y los usos de puntajes o porcentajes de las pruebas como explicación aclarativa, por último, no se descarta la evaluación para demostración representativa". Por eso, es utilizada en la investigación, porque, son las características que se encuentran enmarcadas al contexto educativo estudiado; destacando la coherencia, la síntesis, el análisis, y priorizando las dimensiones expuestas.

La confiabilidad del instrumento se obtuvo, mediante, el Programa Estadístico SPSS con un Coeficiente de Cronbach de 0.886 que comprueba la validez del mismo, puesto que, se realizó la estadística pertinente de los valores obtenidos y, de igual forma, las tabulaciones de cada dimensión relacionadas a las preguntas para determinar el nivel aceptable. Si quien aplica una prueba pretende tomar decisiones sobre una persona, ya sea, en un diagnóstico psicológico o una licencia para ejercer una profesión, etc., se requiere un valor de alta confiabilidad ( 0.90 como mínimo aceptable y 0.95 como la norma deseable); si, en cambio el uso del instrumento es para investigación se requiere una confiabilidad moderada del 0.80 considerada adecuada. (Nunnally, Bernstein, \& Hongan, 2004, pág. 112)

Los datos se procesaron en el Programa Estadístico SPSS, sus valores se analizan con medidas de tendencia central divididas en media, mediana, moda y la desviación estándar, por lo que existe la oportunidad de obtener intervalos para determinar el grado de cumplimiento de cada indicador del instrumento presentado en los resultados y discusión.

\section{RESULTADOS Y DISCUSIÓN}

Los gráficos que se presentan a continuación, expresan los porcentajes en los rangos: débil, intermedio y alto de la encuesta aplicada a los estudiantes del 2do año de BGU de 
la Unidad Educativa "Jhon F. Kennedy" con el propósito de diagnosticar el nivel de comprensión lectora en los educandos. Para ello, se estableció un banco de preguntas semiestructuradas asociadas a tres dimensiones: Comprensión lectora, Técnicas de lectura; y, el tiempo de ocio orientado a la lectura, donde se obtuvieron los siguientes resultados:

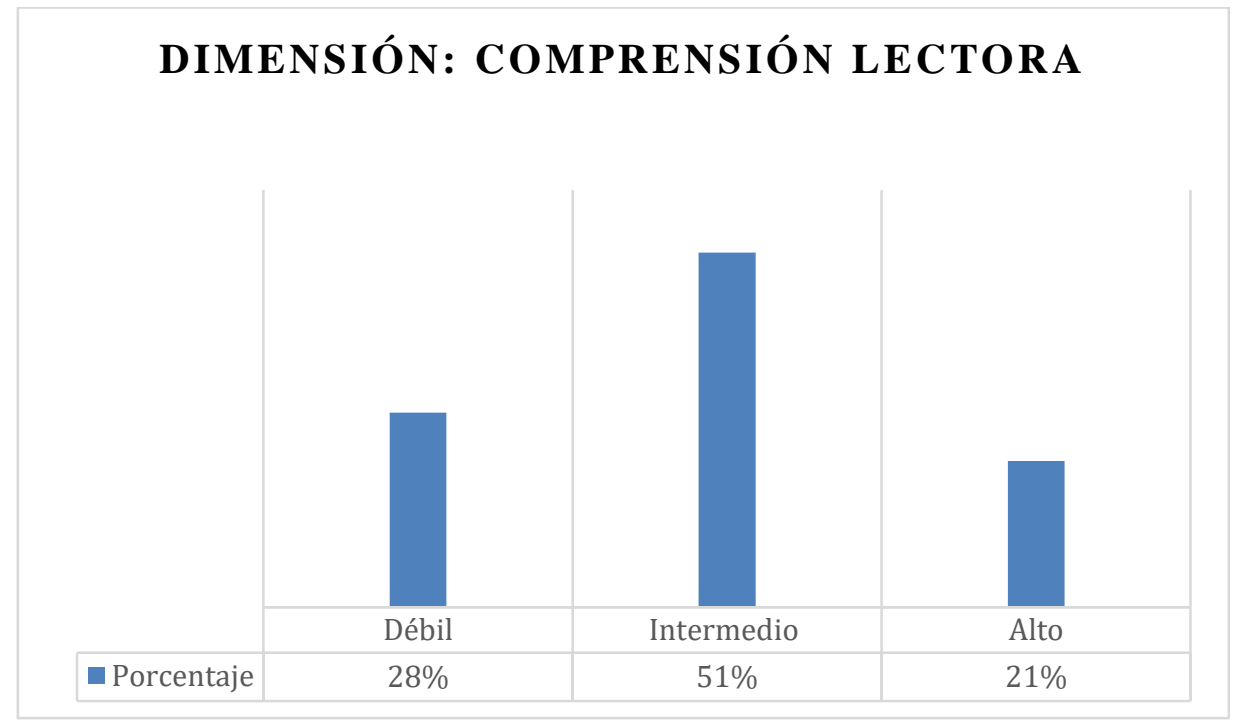

Nota: Encuesta a los estudiantes de la "Unidad Educativa Jhon F Kennedy"

Se observa en la dimensión $\mathrm{N}^{\circ} 1$, que del total de 43 estudiantes encuestados el $28 \%$ muestra un nivel débil en la comprensión de textos; por diferentes motivos, entre ellos, porque no dedican tiempo a la lectura y tienen poco interés al momento de realizar la abstracción e interpretación apropiada de un texto. El resultado reflejo falta de disposición en las prácticas literarias, según afirma Serafini (1991), “el discente pasa horas frente a un libro distraído y desganado o lee y relee el mismo párrafo durante horas intentando aprenderlo de memoria sin lograr comprender su significado". Por ende, no desarrolla un aprendizaje autónomo y mucho menos dedica tiempo en repasos gramaticales para crear nuevos conocimientos que son dependientes de la asimilación, crítica coherente y profundización o argumentación de contenidos como beneficio personal, sino que, se rigen a las directrices y normas del docente.

El $51 \%$ de estudiantes se encuentra en el rango intermedio, esto quiere decir, que existe un interés por desarrollar la competencia lectora, según afirma Ferreiro (2006), "leer representa una necesidad que se debe poner en práctica, porque aportará para la formación académica e integral en los discentes”. Por ello, es una tarea indispensable para la 
formación del educando, puesto que, impulsa la actividad durante la adquisición de nuevas enseñanzas; es así, como los hábitos de lectura forman parte esencial del contexto educativo, siendo necesarios para el desarrollo cognitivo.

El proceso de la práctica lectora contribuye al desarrollo de comprensión e interpretación del material escrito, es importante en la formación de los estudiantes, debido a que, por medio de éste se generan nuevas estructuras cognoscitivas que aumentan la capacidad de raciocinio del discente. Como menciona Bayona (2005), "la importancia de formar hábitos de lectura permite desarrollar la posibilidad y mejorar las habilidades cognitivas que enriquecen el intelecto y favorecen al rendimiento académico", es decir que, leer se describe como un método fundamental para el progreso y también, el crecimiento personal educativo.

El 21\% de los estudiantes encuestados presentan un nivel alto de comprensión lectora. Según, afirma la UNESCO (2016), "la comprensión lectora es la forma que se tiene para acceder a los conocimientos, a la participación activa en la sociedad, dado que se vive en un mundo letrado cada vez más complejo". Es decir, que los educandos sí dedican tiempo para leer y logran entender ya sea el texto completo o partes del mismo, por ejemplo: la introducción, el resumen, las conclusiones, entre otros aspectos literarios. Debido a que, se implican diferentes procesos de forma independiente como: la disposición de espacio, tiempo, interés sobre un libro, tomar en cuenta las ideas principales como, también, secundarias, reorganizar información a través de bosquejos, sintetizar resúmenes, leer en voz alta o en silencio, reconocer palabras técnicas, etc. relacionándolos a las prácticas habituales.

La educación tiene un papel fundamental porque es el factor más directo e inmediato que determina las orientaciones de la lectura en los estudiantes y en la sociedad, puesto que, de ella depende su enseñanza, desarrollo y consolidación. Como menciona Giraldoni (2006), "El hábito de un discente lector se entiende como la acción de realizar la lectura en forma motivada y se considera como medio de aprendizaje y adquisición de nuevos conocimientos". Por ende, todo aporte, es necesario, como refuerzo para la comprensión de un texto y es aquí donde se hacen presentes varias habilidades o destrezas que los discentes adquieren durante el enriquecimiento lingüístico vocalizado, relacionando de forma analítica la información adquirida de un contenido con los saberes previos. 


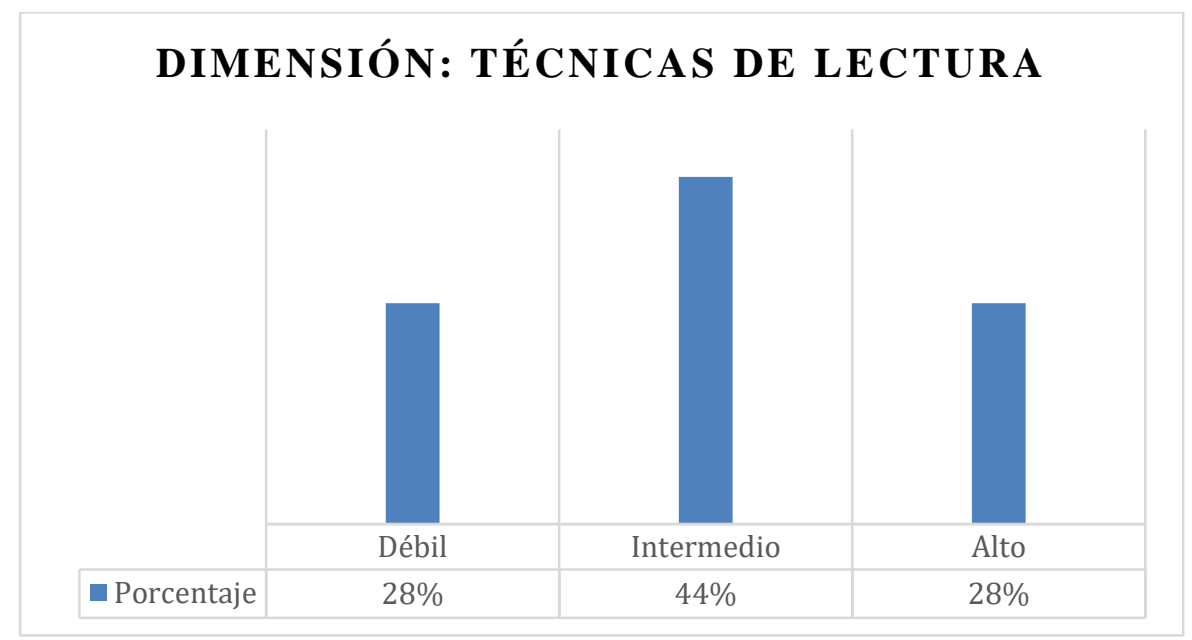

Nota: Encuesta a los estudiantes de la "Unidad Educativa Jhon F Kennedy"

La dimensión $\mathrm{N}^{\circ} 2$ expresa que el $28 \%$ de los estudiantes presentan un nivel débil en el uso de las técnicas de lectura, por tanto, es un porcentaje de encuestados que no logran utilizarlas, de manera, correcta, las mismas que ayudan al desarrollo intelectual, pero se necesita mejorar. De acuerdo con Smith (1990), "los docentes de educación enseñan diversas técnicas en el proceso literario, permitiendo a los discentes comprender, analizar, críticamente textos de diferentes géneros discursivos". Por ello, los resultados demuestran que no realizan esta acción porque los maestros siguen reglas o metodologías para forzar en los educandos leer lo que consideren útil y beneficioso para cumplir cada exigencia de las asignaturas; no se enfocan en las técnicas conducidas al fortalecimiento de destrezas como base de los saberes.

El $44 \%$ de estudiantes muestran un nivel intermedio de interés al emplear las técnicas de lectura, siendo factible para acrecentar sus habilidades o aprendizajes desarrollados sobre un texto; según Martinez (1982), "trata de profundizar y extender el conocimiento del educando, además, de motivar e impulsar su uso". Es decir, que es necesario, que el discente se enfoque en la interpretación inferencial, y la realice, de manera pausada y correcta para obtener un mejor resultado de análisis acorde a un argumento específico, dado que es favorable, utilizar esquemas o mapas mentales para separar, comparar y relacionar conceptos, identificar una secuencia narrativa en la lectura compresiva lo que permite deducir el significado de las palabras en relacion al contexto; para asi, llegar a fortalcer, mediante, en estas técnicas las capacidades congitivas enfocadas en la lectura. El 28\% de estudiantes muestra un nivel alto en el uso de técnicas de lectura, de acuerdo con Bruer (1993) "El promedio eficiente de lectura de unas 25 páginas en una hora, 
transmite un ritmo adecuado, pausado y deducible que puede considerarse como recomendable para estudiantes de bachillerato". Por ende, los discentes si dedican espacios o rutinas para instruirse y desarrollan la lectura comprensiva e interpretativa con la ayuda de herramientas virtuales, por ejemplo: Planet Lector, que es uno de los modos que tienen las Instituciones para organizar las lecturas, por tanto, es evidente que los educandos aborden temas fáciles o complejos y muestren interés por adaptar técnicas de lectura específicas; como leer en diferentes tonalidades o cambios de velocidad lingüística sobre un escrito, por ello, consultan diferentes libros o sitios web como actualización de conocimientos y aprendizajes duraderos.

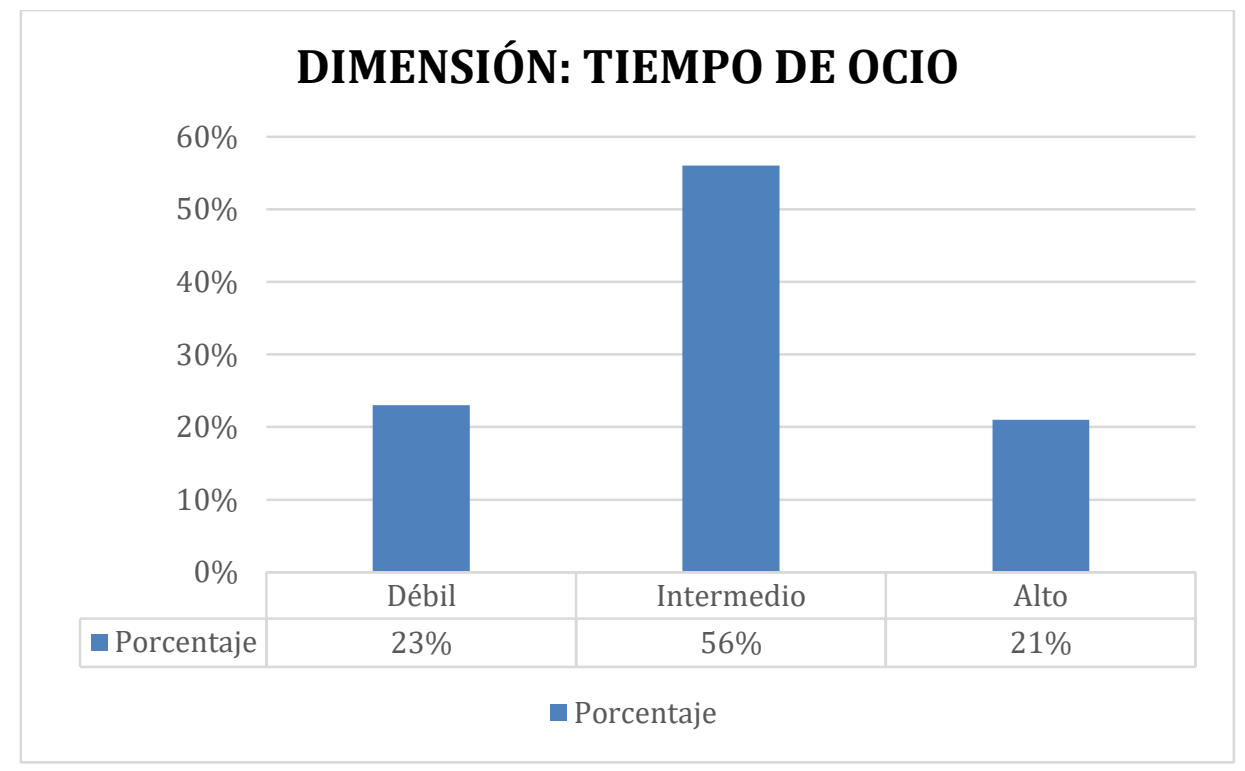

Nota: Encuesta de estudiantes de la "Unidad Educativa Jhon F Kennedy"

La dimensión 3 expresa que el 23\% representa un nivel de interés débil hacia el total del tiempo de ocio orientado a la lectura; esto se debe a lo mencionado por Sandoval (2016) "las actividades de ocio de mayor preferencia por los estudiantes es navegar por Internet, escuchar música y compartir con los amigos, etc." Es decir, se dedica poco tiempo a la lectura, debido a que, los estudiantes no conocen el significado que tienen las palabras, los conceptos de un texto, lo que le da la facilidad para desarrollar la capacidad de pensar, permitiéndole mejorar el vocabulario y comprender ciertos argumentos en el desarrollo de las actividades académicas que, diariamente, realizan.

El 56\% de estudiantes muestran un nivel intermedio de interés orientado a la lectura en tiempo de ocio; señala (Del valle , 2012) “que para adquirir una buena comprensión, es recomendable planificar hora y media de lectura diaria en las Instituciones", por ende, se 
desarrolla el aprendizaje, estructurando percepciones de actividades lectoras, además, se considera importante la recreación del estudiante permitiéndole insertarse en la práctica de alguna actividad de ocio que esté involucrada con la misma, de tal manera que, le permita ampliar sus capacidades cognitivas.

El $21 \%$ de estudiantes se sitúan en el rango alto, es decir, existe una cantidad favorable de estudiantes que aprovechan el tiempo en desarrollar actividades para fomentar la lectura o enriquecer su aprendizaje. De acuerdo con Yubero \& Larrañaga ( 2012), "plantean que para ser lectores se debe leer y esto implica dedicar el propio tiempo de ocio". Por lo que, se considera que al estudiar el discente construye un significado, distribuyendo su espacio libre en repasos de textos, artículos, revista y otros, requiriendo esfuerzo y compromiso para aumentar sus conocimientos, capacidades y habilidades entendiendo y captando todo lo que lee.

\section{CONCLUSIÓN O CONSIDERACIONES FINALES}

La comprensión lectora es una competencia que puede ser desarrollada en los contextos educativos y contribuye a mejorar los procesos cognitivos como: la atención, la imaginación, la creatividad y la interpretación. Ponerla en práctica ayuda a los discentes a resolver interrogantes a procesar información a generar argumentos a mejorar la velocidad, la calidad y la fluidez de la lectura e incrementa el vocabulario y el razonamiento abstracto.

En la primera dimensión, el $21 \%$ de los discentes presentan un nivel alto de comprensión lectora, el $51 \%$ un nivel intermedio y, el $28 \%$ un nivel bajo. Esto indica que, un porcentaje significativo de estudiantes necesita fortalecer la competencia lectora, mediante, hábitos adecuados de lectura que favorezcan el desarrollo de habilidades cognoscitivas que coadyuven en su proceso formativo.

En la segunda dimensión, solo el $28 \%$ de los estudiantes aplican de forma óptima las técnicas de lectura, lo cual, les permite entender los temas centrales del texto, mediante, el uso de mapas mentales, el subrayado y los señalamientos; de igual forma, esto sugiere, que una de las razones por las que los estudiantes no desarrollan la competencia lectora se debe a la falta de aplicación de estas técnicas. Así mismo, en la tercera dimensión, solo el $21 \%$ de los estudiantes durante el ocio dedican más tiempo a la lectura. 
Por tanto, es fundamental que, dentro de la Institución Educativa en estudio, los docentes promuevan el interés hacia la lectura y que los estudiantes palpen por sí mismos sus beneficios en su formación académica y personal, ya que, es a través de la comprensión lectora que los discentes fortalecen sus capacidades como lectores y desarrollan sus facultades cognoscitivas

\section{LISTA DE REFERENCIAS}

Alvarez Alberdi, C. M. (1993). Técnicas de lectura eficaz. Dialnet, 83-91.

Armella, V. A., \& López De Llergo, A. T. (2004). Hacia un desarrollo humano: valores, actitudes y hábitos. México: Limusa.

Bayona, V. M. (2005). Lectores competetentes. Revista de educación, 15.

Bruer, J. (1993). Escuelas para pensar. Una ciencia del aprendizaje en el aula. Redalyc.

Cruz. (2004). Acerca de la lectura. Madrid: Palibrio.

Del valle , M. J. (2012). Variables que inciden en la adquisición de hábitos de lectura de los estudiantes. Guatemala: Digeduca .

Díaz, \& Hernández. (2010). Actitudes, hábitos y estrategias de lectura de ingresantes a la educación superior. Colombia: Redalyc "Educación y Educadores".

Ferreiro, E. (2006). La escritura antes de la letra. Veracruz, Mexico: redalyc.org.

García, M. S. (2013). Lectura, ocio y rendimiento académico en estudiantes. Scielo, 133.

Giraldoni, C. (2006). Lectura y análisis cuali-cuantitativo del uso, accesibilidad y valoracion de los libros. calidad de la educación, 25.

Gutierrez Braojos, C., \& Salmerón Pérez, H. (2012). Estrategias de comprensión lectora: enseñanza y evaluación en la educación primaria. Redalyc, 183-202.

Hernández Sampieri, R. (2003). Metodología de la investigación. México: Editorial Mexicana.

Hoyos Flóres, A. M., \& Gallegos, T. M. (2017). Desarrollo de habilidades de compresión lectora en niños y niñas de la básica primaria. Revista Virtual de la Universidad Católica del Norte, 23-27.

Jiménez Pérez, E. (2014). Compresión lectora vs competencia lectora: qué son y qué relación existe entre ellas. Redalyc, 65-74.

Kane, M. T. (2006). Validación de interpretaciones y usos de partituras. American Council on Education and Praeger Publishers. 
León, O. G., \& Montero, I. (2003). Métodos de investigación en Psicología y Educación ( $3^{a}$ ed.). Madrid: McGraw-Hill: Redalyc.

Luque. (2005). Estrategias de aprendizaje en comunicación integral. Madrid: Palibrio.

Madero Suárez, I. P., \& Gómez López, L. F. (2013). Proceso de comprensión lectora en alumnos de 3ro de secundaria. Revista Mexicana de Investigación Educativa, 2021.

Mantilla. (2008). Estrategias para fomentar el gusto y el hábito de la lectura. Bogotá.

Martinez, M. (1982). Tipos de seminario en la educación superior. Redalyc.

Muñoz. (2002). Identidad e identificación: investigación de campo como herramienta de aprendizaje. Dialnet, 13.

Nunnally, Bernstein, \& Hongan. (2004). Metodología de la investigación: confiabilidad. Dialnet, 112.

Rivas Cedeño, L. (2015). Metodología para el Desarrollo de la comprensión lectora. Dialnet, 47-61.

Sabino, C. (1992). Conceptos del estudio descriptivo. Bogóta: Lumen, Buenos Aires.

Sandoval, N. (2016). Diágnostico acerca del uso de ocio y el tiempo libre entre los estudiantes de la Universidad Nacional experimental de Táchira. Pedagogia Social, 169-188.

Serafini. (1991). Métodos, preferencias y hábitos de lectura en estudiantes de pregrado. Revista Teoría y Praxis Investigativa, 3(1).

Smith, F. (1990). Para darle sentido a la lectura. Aprendizaje Visor.

Snow. (2001). Reading for understading. Redalyc.

Solé, I. (1997). La lectura un proceso estratégico. Barcelona: Graó.

Solé, I. (1998). Estrategias de lectura. Barcelona: Graó.

Solé, I. (2012). Competencia lectora y aprendizaje. Iberoamericana de Educación, 43.

Suárez, M. L. (2002). Lectura para el aprendizaje. Publicación Juvenil Latinoamericana, 20-21.

Tello, J. G. (2001). Tiempo de ocio en estudiante de colegios fiscales. Buen Lector, 12424.

Tovar Cabañas, R. (2009). Técnicas, tipos y velocidades de lectura tras la investigación documental. Redalyc, 38-78. 
Diagnóstico de la compresión lectora...

UNESCO. (2016). Sobre lectura, hábito lector y sistema educativo. Distrito Federal, México: Redalyc.

Yubero, S., \& Larrañaga, E. (2012). El valor de la lectura en relación con el comprensión lectora. EDUCA, 20. 